\title{
A Three-Phase Grid Synchronization System with Sub and Interharmonics Immunity
}

\author{
Renato Guerreiro Araújo ${ }^{1}$, Francisco Kleber A. Lima ${ }^{1}$, Ernande Eugênio C. Morais ${ }^{1}$, Carlos Gustavo C. Branco ${ }^{1}$, \\ Juliana Isabel L. Uchôa ${ }^{1}$, and José de Ribamar L. Filho ${ }^{2}$ \\ ${ }^{1}$ Department of Electrical Engineering \\ Federal University of Ceara, UFC \\ Fortaleza, Ceara, Brazil
}

Phone/Fax number: +0055 853366 9413, e-mail: renato.g.a@hotmail.com, klima@dee.ufc.br, ernande@alu.ufc.br, gustavo@dee.ufc.br, juliana@dee.ufc.br

${ }^{2}$ Eletrobras Distribution Piaui

Research Management, Development and Energy Efficiency

Teresina, PI, Brazil

e-mail: ribamarlima@eletrobraspiaui.co

\begin{abstract}
This paper discusses a new PLL (Phase- LockedLoop) approach for detection of the fundamental positivesequence component of three-phase systems. The structure presented is based on the correlation of the input signal with a complex signal generated from the use of an adaptive filter in a PLL algorithm. Moreover, the adapted PLL, due to the use of the adaptive filter, presents a higher level of rejection for two particular disturbances: interharmonic and subharmonic, when compared to the original algorithm. Simulation and experimental results will be presented in order to prove the efficacy of the proposed adaptive algorithm. Several scenarios will be presented, to which the algorithm will be exposed. The response of the algorithm also be compared to the synchronization system based on DSOGI-FLL.
\end{abstract}

\section{Key words}

Adaptive Filter, Interharmonics, Phase-Locked Loop, Subharmonics, Synchronization System.

\section{Introduction}

The detection of fundamental positive-sequence components of voltages and/or currents has a great importance for the control strategy of three-phase gridconnected power converters [1] [2]. The information generated by the synchronization system is used at different levels in the control strategy of the power converters connected to the grid. The positive sequence data can be employed for power calculations, alignment of vector control techniques, among others relevant tasks. Specifically, converter-interfaced distributed generation (DG) requires a proper synchronization under grid disturbances [3]. The positive-sequence detection systems are being widely studied, mainly using closed-loop adaptive algorithms in order to get proper accurate synchronization signals [2] [3] [4].
When the input signal is characterized as a pure sine wave, a zero crossing detector performs this task satisfactorily. However, due to the presence of harmonics in the input signal, the detection method and other methods derived of this do not provide, in general, satisfactory results [5] [6]. Most recently, different methodologies for synchronous phase-angle detection based on PLL (Phase-Locked Loop) have been proposed and analyzed both in terms of dynamic response and steady-state error [7] [8] [9] [10]. Usually, input signals used to obtain the synchronization reference are distorted. In addition, for three-phase system, these voltage and/or current can also be unbalanced. Therefore, the synchronization system performance must have a low sensitivity to these disturbances.

The presence, in particular, of subharmonics and interharmonics cause a reduction of accuracy in particular synchronization methods based on PLL, due to the sensitivity of these methods to these disturbances. As increases the amplitude of the subharmonic and of the interharmonics there is an increase in the error of the values estimated by the synchronization system. This could cause the wrong operation of power converters, active filters and reading mistakes of measuring instruments that work with the measurement of synchronized signals [11] [12] [13].

In particular, there are two basic mechanisms for generating subharmonics and interharmonics. The first mechanism is the generation of these disturbances due to fast variation of current in the equipments and installations. These variations are generated by loads operating in transitional arrangements. The second mechanism can be determined as the switching (do not synchronized with the frequency of the power system) of semiconductor devices in static converters. The main sources of these disturbances are loads that use electric arc, electric drives of variable load and static converters [14]. From to the exposed, this paper aims to provide a threephase PLL algorithm that have high rejection capability of 
interharmonics and subharmonic. This synchronization system can be used quite effectively in more intelligent control strategies for power converters. The proposed synchronization system will be evaluated by means of mathematical analysis and computer simulations performed using the PSCAD/EMTDC software. Will be analyzed aspects such as the disturbance rejection capability and dynamic response of the algorithm. In order to perform the comparative analysis, the algorithm will be compared with the synchronization system based on the DSOGI-FLL. Finally, experimental results obtained through HIL (Hardware-in-the-Loop) platform are presented, which will be compared to the simulations results obtained in order to validate the proposed simulation study.

\section{A Three-Phase PLL Algorithm With Harmonic Distortion Immunity}

The structure of the three-phase PLL proposed in [15] is shown in Figure 1. This structure considers the voltage or current present in a three-phase system and from of these quantities it is able to estimate the fundamental positive sequence component of the input signal $\boldsymbol{y}_{\mathbf{1}}^{+}(\mathbf{t})$, the synchronization angle $\widehat{\boldsymbol{\theta}_{1}^{+}}(\mathbf{t})$, the frequency $\widehat{\mathbf{f}_{\mathbf{1}}}$, and the fundamental positive-sequence component of the input signal $\mathbf{u}(\mathbf{t})$.

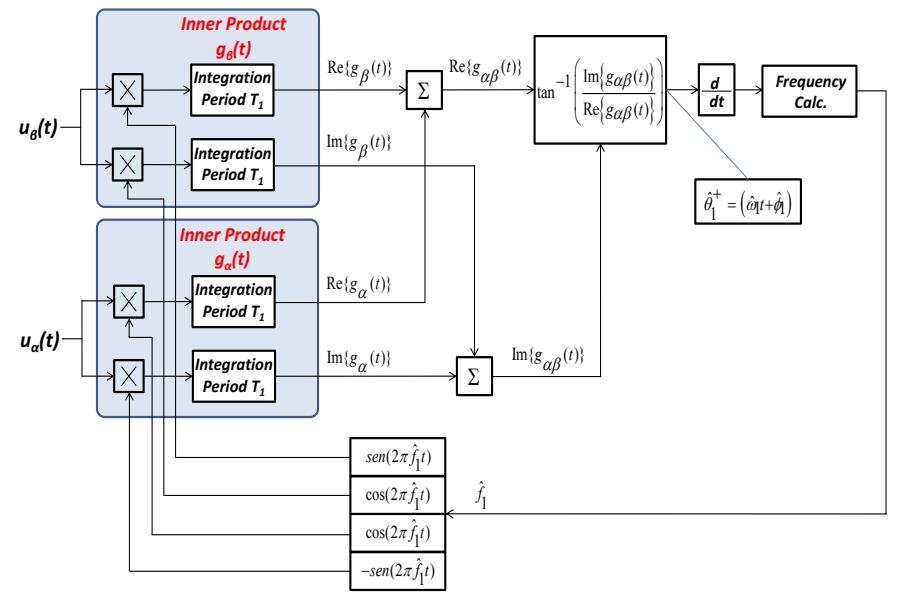

Fig. 1. Structure of the Three-phase PLL.

The phase-voltages in abc reference frame are converted to the $\alpha \beta$ reference frame and feed the PLL algorithm inputs. The detector uses the $\alpha \beta$ components to obtain an estimative of the fundamental frequency, which is in turn used to estimate the amplitudes and phase-angles of the fundamental positive sequence components.

The input signal (in $\alpha \beta$ reference frame) can be represented by (1):

$$
u_{\alpha \beta}(t)=\left[\begin{array}{c}
u_{\alpha}(t) \\
u_{\beta}(t)
\end{array}\right]=\left[\begin{array}{c}
V_{1} \times \operatorname{sen}\left(2 \pi f_{1} t+\phi_{1}\right) \\
V_{1} \times \operatorname{sen}\left(2 \pi f_{1} t+\phi_{1}-\pi / 2\right)
\end{array}\right] .
$$

From the correct estimation of the input voltage fundamental component frequency, $\widehat{\mathbf{f}_{\mathbf{1}}}=\mathbf{f}_{\mathbf{1}}$, it is possible to generate a three-phase complex subspace of the signals. The complex subspace on the $\alpha \beta$ reference frame is given by (2):

$$
\left\{\begin{array}{l}
e^{-j\left(2 \pi \hat{f}_{1} t\right)}=\cos \left(2 \pi \hat{f}_{1} t\right)-j \operatorname{sen}\left(2 \pi \hat{f}_{1} t\right) \\
e^{-j\left(2 \pi \hat{f}_{1} t-\pi / 2\right)}=\cos \left(2 \pi \hat{f}_{1} t-\pi / 2\right)-j \operatorname{sen}\left(2 \pi \hat{f}_{1} t-\pi / 2\right)
\end{array}\right.
$$

By making the projection of the signals (1) on (2), one can obtain the synchronization angle $\left(\widehat{\boldsymbol{\theta}_{1}^{+}}\right)$of the fundamental positive sequence component of the input signal. The vector of inners-products $\vec{g}_{\alpha \beta}(t)$ is given by (3):

$$
g_{\alpha \beta}(t)=\left[\begin{array}{l}
g_{\alpha}(t) \\
g_{\beta}(t)
\end{array}\right]=\left[\begin{array}{c}
\int_{T_{1}} u_{\alpha}(t) \times e^{-j\left(2 \pi \hat{f}_{1} t\right)} d t \\
\int_{T_{1}} u_{\beta}(t) \times e^{-j\left(2 \pi \hat{f}_{1} t-\pi / 2\right)} d t
\end{array}\right] .
$$

The inner product will generate a real and imaginary signals relating to each coordinate of the reference frame $\boldsymbol{g}_{\boldsymbol{\alpha}}(\boldsymbol{t})$ and $\boldsymbol{g}_{\boldsymbol{\beta}}(\boldsymbol{t})$. The is comprising a real part $\boldsymbol{R} \boldsymbol{e}\left\{\overrightarrow{\boldsymbol{g}}_{\boldsymbol{\alpha} \boldsymbol{\beta}}(\boldsymbol{t})\right\}$ and imaginary part $\boldsymbol{I m}\left\{\overrightarrow{\boldsymbol{g}}_{\boldsymbol{\alpha} \boldsymbol{\beta}}(\boldsymbol{t})\right\}$. The real and imaginary part are given by (4):

$$
\left[\begin{array}{c}
\operatorname{Re}\left\{g_{\alpha \beta}(t)\right\} \\
\operatorname{Im}\left\{g_{\alpha \beta}(t)\right\}
\end{array}\right]=\left[\begin{array}{l}
\operatorname{Re}\left\{g_{\alpha}(t)\right\}+\operatorname{Re}\left\{g_{\beta}(t)\right\} \\
\operatorname{Im}\left\{g_{\alpha}(t)\right\}+\operatorname{Im}\left\{g_{\beta}(t)\right\}
\end{array}\right] .
$$

From (3) and (4) one can obtain the real part of each coordinate according to (5) and the imaginary part can be obtained similarly according to (6):

$$
\begin{gathered}
\left\{\begin{array}{l}
\operatorname{Re}\left\{g_{\alpha}(t)\right\}=\int_{T_{1}} u_{\alpha}(t) \times \cos \left(2 \pi \hat{f}_{1} t\right) d t ; \\
\operatorname{Re}\left\{g_{\beta}(t)\right\}=\int_{T_{1}} u_{\beta}(t) \times \operatorname{sen}\left(2 \pi \hat{f}_{1} t\right) d t .
\end{array}\right. \\
\left\{\begin{array}{c}
\operatorname{Im}\left\{g_{\alpha}(t)\right\}=-\int_{T_{1}} u_{\alpha}(t) \times \operatorname{sen}\left(2 \pi \hat{f}_{1} t\right) d t ; \\
\operatorname{Im}\left\{g_{\beta}(t)\right\}=\int_{T_{1}} u_{\beta}(t) \times \cos \left(2 \pi \hat{f}_{1} t\right) d t .
\end{array}\right.
\end{gathered}
$$

From the signals $\boldsymbol{R} \boldsymbol{e}\left\{\overrightarrow{\boldsymbol{g}}_{\boldsymbol{\alpha} \boldsymbol{\beta}}(\boldsymbol{t})\right\}$ and $\operatorname{Im}\left\{\overrightarrow{\boldsymbol{g}}_{\boldsymbol{\alpha} \boldsymbol{\beta}}(\boldsymbol{t})\right\}$ resulting from the inner product $\overrightarrow{\boldsymbol{g}}_{\boldsymbol{\alpha} \boldsymbol{\beta}}(\boldsymbol{t})$ can be obtained the synchronism angle of the fundamental positive sequence of the input signal $\left.\overrightarrow{\boldsymbol{u}}_{\boldsymbol{\alpha} \boldsymbol{\beta}}(\boldsymbol{t})\right\}$ according to (7):

$$
\hat{\theta}_{1}^{+}(t)=\tan ^{-1}\left(\frac{\operatorname{Im}\left\{g_{\alpha \beta}(t)\right\}}{\operatorname{Re}\left\{g_{\alpha \beta}(t)\right\}}\right)=\left(2 \pi \hat{f}_{1} t+\hat{\phi}_{1}^{+}\right)
$$

From the synchronization angle information $\widehat{\boldsymbol{\theta}}_{\mathbf{1}}^{+}$is obtained the angular frequency $\widehat{\boldsymbol{\omega}}_{\mathbf{1}}$ by the time derivative of the angular displacement $\widehat{\boldsymbol{\theta}}_{1}^{+}$. The angular frequency $\widehat{\boldsymbol{\omega}}_{\mathbf{1}}$ is the internal input of the loop that performs the calculation of the fundamental frequency, in $\mathrm{Hz}$, of the input signal. The internal loop, which performs the calculation of the frequency, is illustrated in Figure 2. 


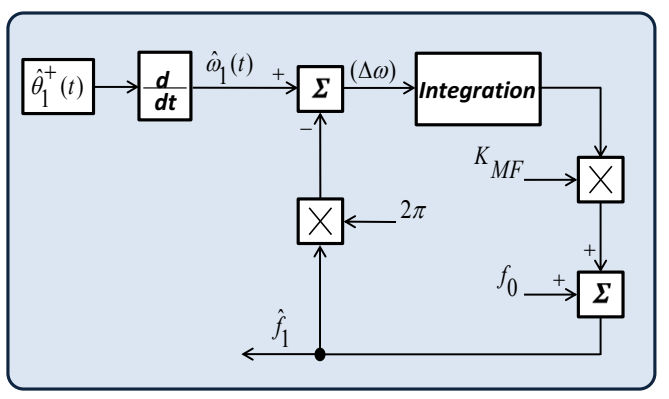

Fig. 2. Internal loop for frequency estimation.

Adopting $\mathbf{f}_{\mathbf{0}}$ as an initial estimative of the frequency, in $\mathrm{Hz}$, the estimated frequency $\widehat{\mathbf{f}_{\mathbf{1}}}$ at any time will be:

$$
\hat{f}_{1}(t)=f_{0}+K_{M F} \int\left(\frac{d}{d t} \hat{\theta}_{1}^{+}(t)-2 \pi \hat{f}_{1}\right) d t
$$

The internal loop frequency estimation performs yet, the paper of a low pass filter with transfer function given by (9) and is represented by the block diagram of Figure 3.

$$
\frac{\hat{f}_{1}(s)}{\hat{\omega}_{1}(s)}=\frac{K_{M F}}{s+2 \pi \cdot K_{M F}} .
$$

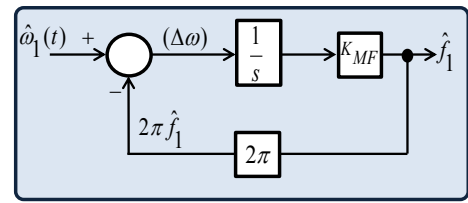

Fig. 3. Block diagram of internal loop for frequency estimation

\section{Optmized Three-Phase PLL Algorithm}

Modifying the structure proposed in [15], it was considered only the calculation process of the real part $\boldsymbol{R} \boldsymbol{e}\left\{\overrightarrow{\boldsymbol{g}}_{\boldsymbol{\alpha} \boldsymbol{\beta}}(\boldsymbol{t})\right\}$ of the inner product $\overrightarrow{\boldsymbol{g}}_{\boldsymbol{\alpha} \boldsymbol{\beta}}(\boldsymbol{t})$ given by (3). The imaginary part $\operatorname{Im}\left\{\overrightarrow{\boldsymbol{g}}_{\boldsymbol{\alpha} \boldsymbol{\beta}}(\boldsymbol{t})\right\}$ is estimated by an adaptive filter in which its input is the signal $\boldsymbol{R} \boldsymbol{e}\left\{\overrightarrow{\boldsymbol{g}}_{\boldsymbol{\alpha} \boldsymbol{\beta}}(\boldsymbol{t})\right\}$. Thus, there will be two orthogonal signals $\boldsymbol{R} \boldsymbol{e}\left\{\overrightarrow{\boldsymbol{g}}_{\boldsymbol{\alpha} \boldsymbol{\beta}}(\boldsymbol{t})\right\}_{-} \mathbf{f}$ and $\boldsymbol{I m}\left\{\overrightarrow{\boldsymbol{g}}_{\alpha \boldsymbol{\beta}}(\boldsymbol{t})\right\}_{-} \mathbf{f}$ from the input $\boldsymbol{R} \boldsymbol{e}\left\{\overrightarrow{\boldsymbol{g}}_{\boldsymbol{\alpha} \boldsymbol{\beta}}(\boldsymbol{t})\right\}$. This makes the number of mathematical operations are reduced and there is an increase in the interharmonic and subharmonic rejection. The remainder of the algorithm is the same as the original algorithm. Figure 4 illustrates the block diagram of PLL considering the insertion of the adaptive filter.

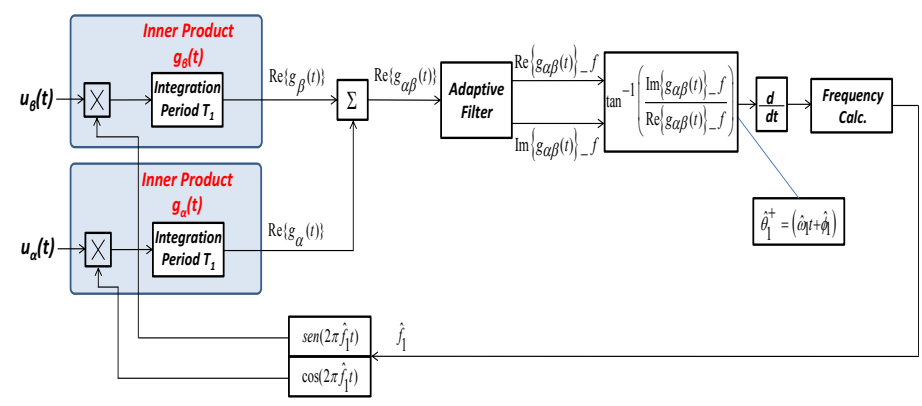

Fig. 4. Block diagram of the optimized Three-phase PLL.

The used adaptive filter, shown in Figure 5, is an adapted version for single-phase systems obtained from a model for three-phase systems analyzed in [16]. The original version is utilized in three phase systems operating in $\alpha \beta$ coordinates. Therefore, it is required the orthogonal signal input obtained after application of the Clarke transformation on the signals in abc coordinates.

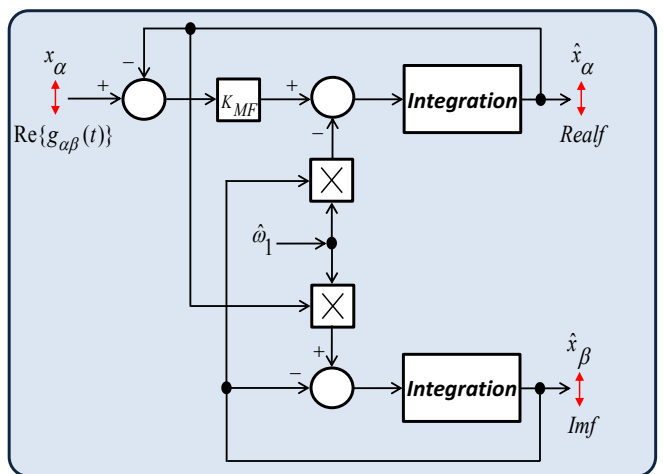

Fig. 5. Block diagram of the adaptive filter.

The original version of the discussed filter can be represented as the following expressions:

$$
\left\{\begin{array}{l}
\hat{x}_{\alpha}(s)=\frac{1}{s}\left[K_{M F}\left(x_{\alpha}(s)-\hat{x}_{\alpha}(s)\right)-\hat{\omega}_{1} \hat{x}_{\beta}(s)\right] \\
\hat{x}_{\beta}(s)=\frac{1}{s}\left[K_{M F}\left(x_{\beta}(s)-\hat{x}_{\beta}(s)\right)+\hat{\omega}_{1} \hat{x}_{\alpha}(s)\right]
\end{array} .\right.
$$

The modified model, for the application of this work, presents the following resulting expressions:

$$
\left\{\begin{array}{l}
\hat{x}_{\alpha}(s)=\frac{1}{s}\left[K_{M F}\left(x_{\alpha}(s)-\hat{x}_{\alpha}(s)\right)-\hat{\omega}_{1} \hat{x}_{\beta}(s)\right] \\
\hat{x}_{\beta}(s)=\frac{1}{s}\left[-\hat{x}_{\beta}(s)+\hat{\omega}_{1} \hat{x}_{\alpha}(s)\right]
\end{array} .\right.
$$

Presenting the following transfer function:

$$
\begin{aligned}
& \frac{\hat{x}_{\alpha}(s)}{x_{\alpha}(s)}=\frac{K_{M F}(s+1)}{s^{2}+s\left(K_{M F}+1\right)+K_{M F}+\hat{\omega}_{1}^{2}} . \\
& \frac{\hat{x}_{\beta}(s)}{x_{\alpha}(s)}=\frac{K_{M F} \hat{\omega}_{1}}{s^{2}+s\left(K_{M F}+1\right)+K_{M F}+\hat{\omega}_{1}^{2}} .
\end{aligned}
$$

Using this idealized filter, quadrature signals are generated from the input signal. These signals are used to form the complex subspace presented in (2) required to get the synchronism angle.

It can be seen that using the adaptive filter, the real and imaginary signals will be less affected by disturbances in relation with input signal $\boldsymbol{R} \boldsymbol{e}\left\{\overrightarrow{\boldsymbol{g}}_{\boldsymbol{\alpha} \boldsymbol{\beta}}(\boldsymbol{t})\right\}$, since there will be an increase in immunity to disturbances, in particular the interharmonic and subharmonic. If on one hand the insertion of the adaptive filter provides an improvement in the rejection of disturbances, on the other hand it is imperative to consider your impact on the dynamics of the algorithm in this situation. In the results, will be exposed the dynamic response of the algorithm facing strongly distorted signals. 


\section{Simulation Results}

To validate the proposed study, simulations were performed in PSCAD/EMTDC software.

\section{A. Steady State Simulation}

Two scenarios were simulated. In the first scenario (Figure 6), the three-phase input signal is composed by the fundamental component, $10 \%$ distortion at $30 \mathrm{~Hz}$ and $10 \%$ distortion at $35 \mathrm{~Hz}$. In the second scenario (Figure 7) the input voltage is composed by the fundamental component, $20 \%$ of distortion at $222 \mathrm{~Hz}$ and $20 \%$ of distortion at 312 $\mathrm{Hz}$.

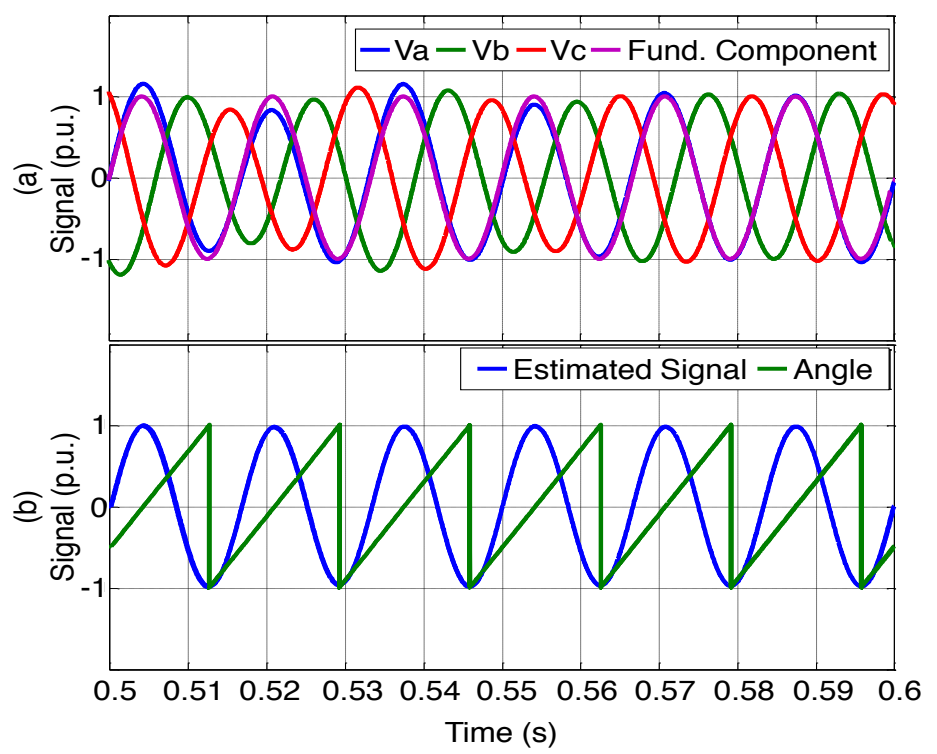

Fig. 6: Performance of the Three-phase PLL for subharmonics: (a) input signal and fundamental component, (b) estimated signal and angle.

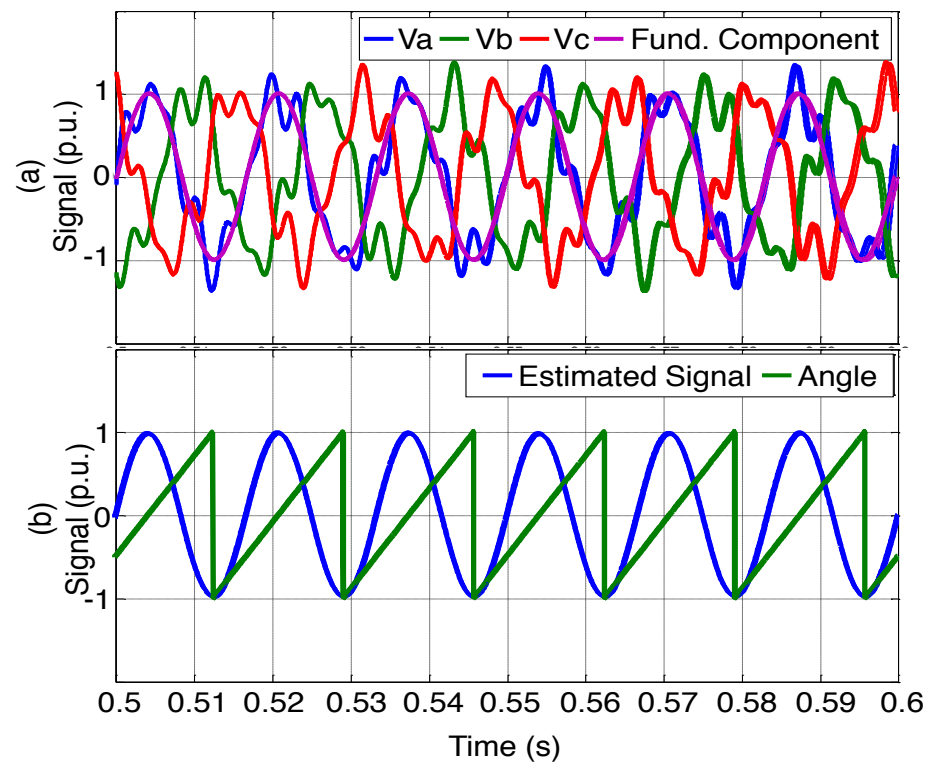

Fig. 7: Performance of the Three-phase PLL for interharmonics: (a) input signal and fundamental component, (b) estimated signal and angle.

It can be seen that even with the strongly distorted input signal, the algorithm obtains satisfactory results.

\section{B. Dynamic Behavior}

In this case, two scenarios were simulated. The input values were considered the same as the presented in Figures 6 (a) and 7 (a) for subharmonics and interharmonics, respectively. In both cases, at time $\mathrm{t}=0.5 \mathrm{~s}$, the amplitude of the fundamental component changes from 1.0 p.u. to 0.8 p.u., the phase changes from $0^{\circ}$ to $30^{\circ}$ and the frequency changes from $60 \mathrm{~Hz}$ to $62 \mathrm{~Hz}$. The Figure 8 presents the estimated values in the presence of subharmonics and the Figure 9 presents the estimated values in the presence of interharmonics.

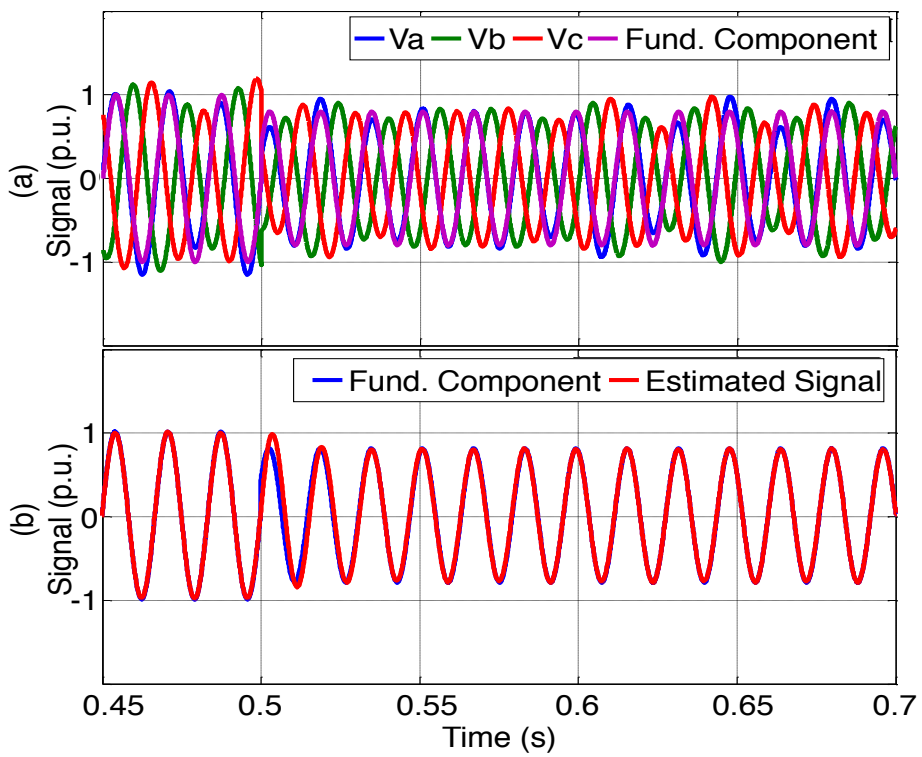

Fig. 8: Dynamic performance for subharmonics: (a) input signal and fund. component, (b) fund. component and estimated signal.

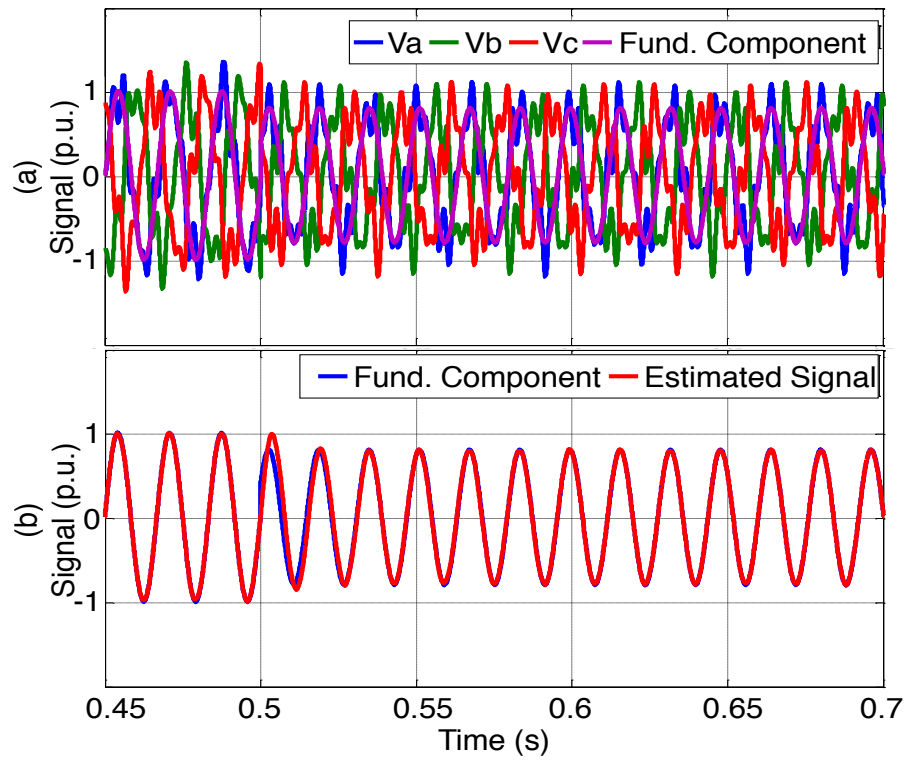

Fig. 9: Dynamic performance for interharmonics: (a) input signal and fund. component, (b) fund. component and estimated signal.

The algorithm obtains satisfactory results. It can be seen that, in both cases, $100 \mathrm{~ms}$ after the time $\mathrm{t}=0.5 \mathrm{~s}$, the estimated output is synchronized with the fundamental component of the input signal. 


\section{Comparison with DSOGI-FLL}

The DSOGI-FLL was adjusted with the gain value 0.5 and the value of $\gamma$ adjusted was 100. Applying the same input signals previously described, it follows that the rms value of the obtained error for subharmonic and interharmonic can be seen in Figure 10 (a) and Figure 10 (b), respectively.

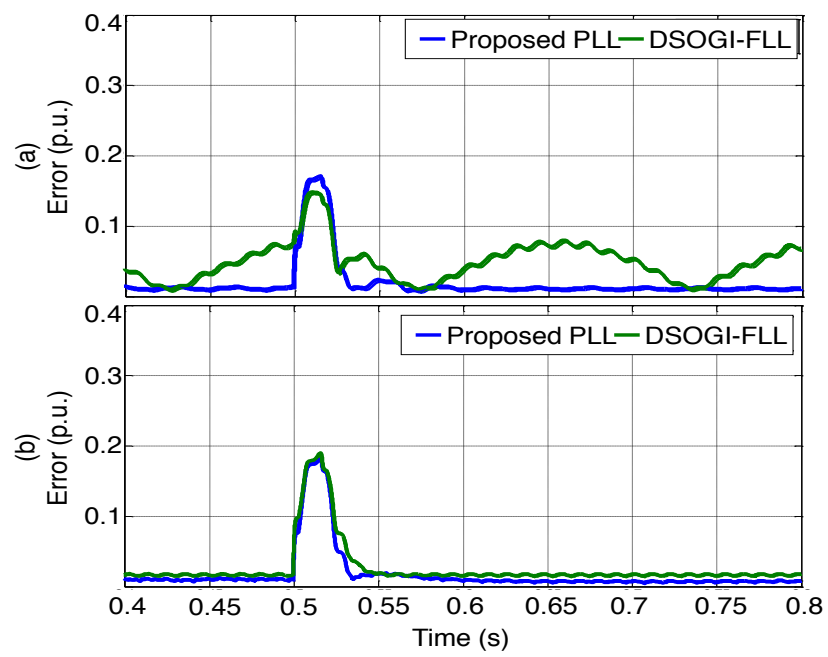

Fig. 10: Comparison of the error between the proposed PLL and the DSOGI-FLL: (a) error in the presence of subharmonics, (b) error in the presence of interharmonics.

Analyzing the behavior of the error in the Figure 10(a), it is observed that the steady state error presented by the proposed PLL is 0.01 p.u. and the settling time is $100 \mathrm{~ms}$. The steady state error presented by the DSOGI-FLL is 0.068 p.u. and the settling time is $60 \mathrm{~ms}$.

Analyzing the behavior of the error in the Figure 10(b), it is observed that the steady state error presented by the proposed PLL is 0.006 p.u. and the settling time is $100 \mathrm{~ms}$. The steady state error presented by the DSOGI-FLL is 0.02 p.u. and the settling time is $60 \mathrm{~ms}$.

Although to present a slower dynamic response, the results define the proposed PLL as another option of synchronization system algorithm in situations where it is desired a finer accuracy as regards the presence of subharmonics and interharmonics.

\section{Experimental Results}

Next are presented the experimental results implemented in the HIL (Hardware-in-the-Loop) platform DSPACE 1103. The Figure 11 presents the input signal, the synchronism angle and the estimated signal for an input signal composed by the fundamental, $10 \%$ of subharmonic at $30 \mathrm{~Hz}$ and $10 \%$ of subharmonic at $36 \mathrm{~Hz}$. The Figure 12 presents the input signal, the synchronism angle and the estimated signal for an input signal composed by the fundamental component, $20 \%$ of interharmonic at $222 \mathrm{~Hz}$ and $20 \%$ of interharmonic at $312 \mathrm{~Hz}$.

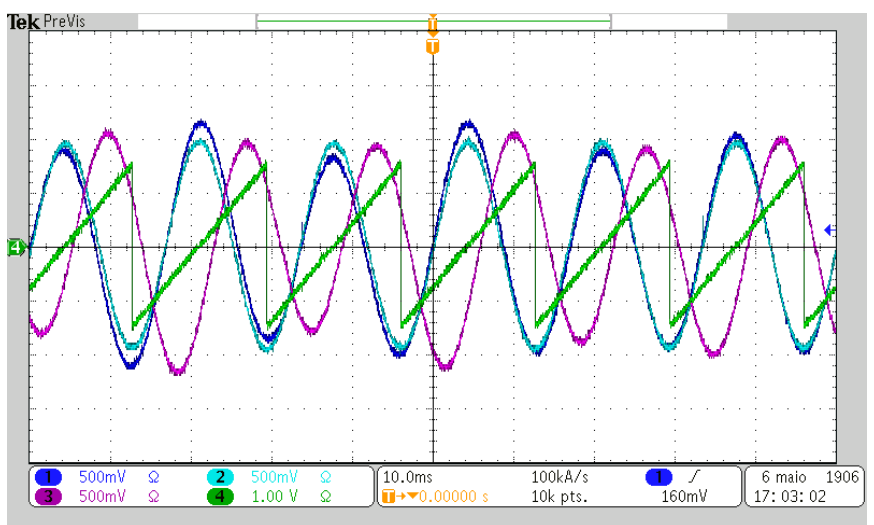

Fig. 11: Input signal, estimated signal and synchronism angle.

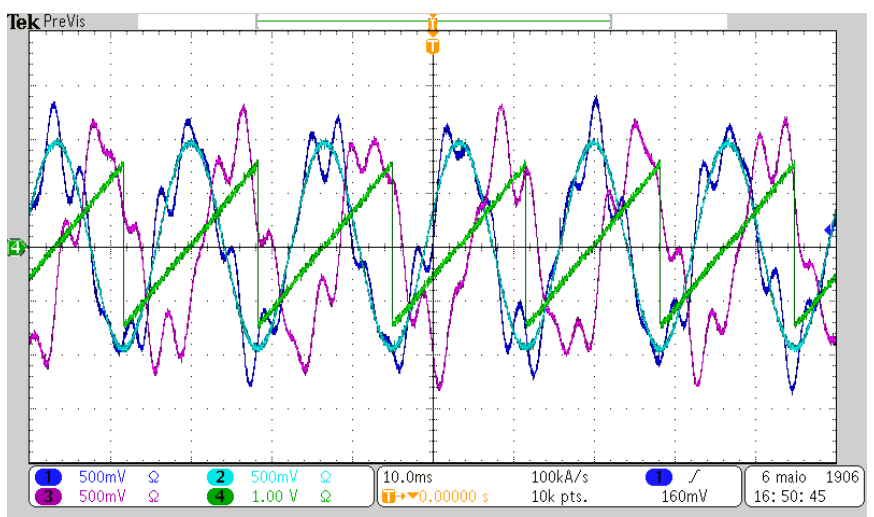

Fig. 12: Input signal, estimated signal and synchronism angle.

Next are analyzed the dynamic response. Two scenarios were implemented. The input values were considered the same as the presented in the Figures 11 and 12 for subharmonics and interharmonics, respectively. In both cases, at time $t$, the amplitude of the fundamental component changes from 1.0 p.u. to 0.8 p.u., the phase changes from $0^{\circ}$ to $30^{\circ}$ and the frequency changes from 60 $\mathrm{Hz}$ to $62 \mathrm{~Hz}$. The Figure 13 presents the estimated values in the presence of subharmonics and the Figure 14 presents the estimated values in the presence of interharmonics.

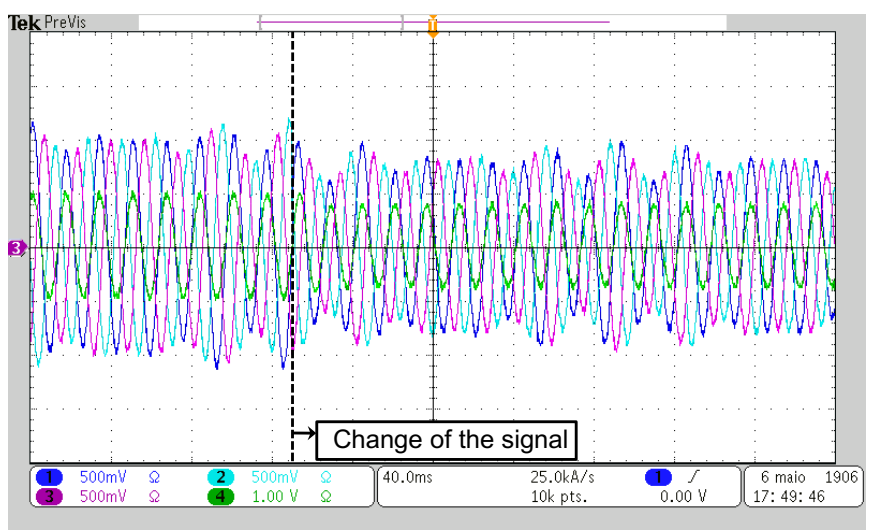

Fig. 13: Input signal and estimated signal. 


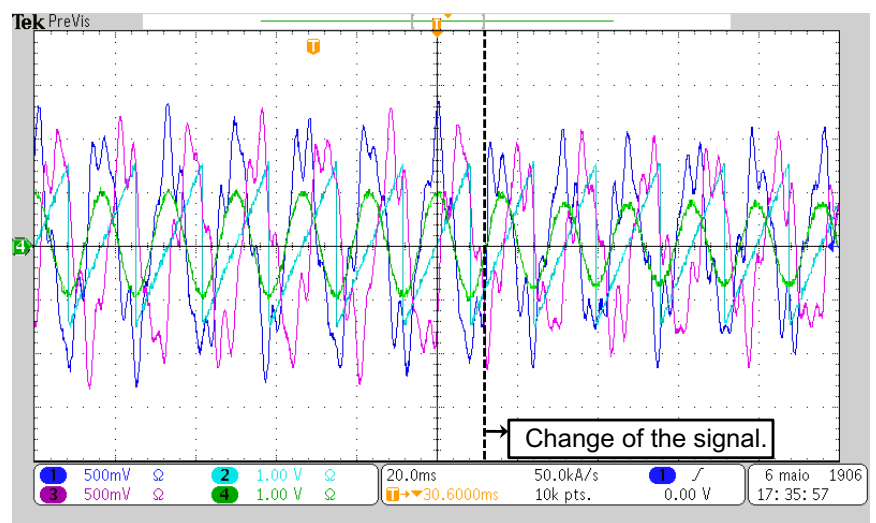

Fig. 14: Input signal, estimated signal and synchronism angle.

To a previously submitted input signal (Figure 13 for subharmonics and Figure 14 for interharmonics), it is presented the rms value of the error generated by the proposed Three-Phase PLL and by the DSOGI-FLL, regarding the fundamental component of the input signal. The Figure 15 presents the error for subharmonics and the Figure 16 presents the error for interharmonics.

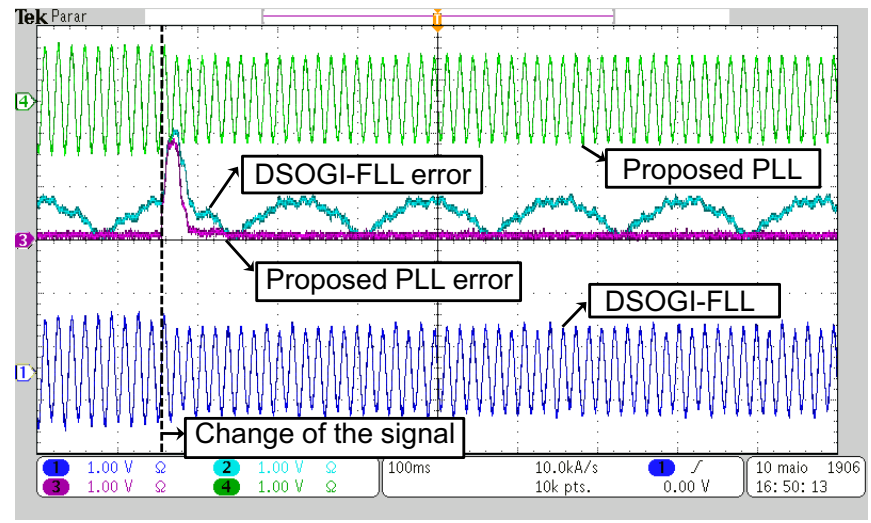

Fig. 15: Signal estimated by the proposed PLL, signal estimated by the DSOGI-FLL, error of the proposed PLL and error of the DSOGI-FLL.

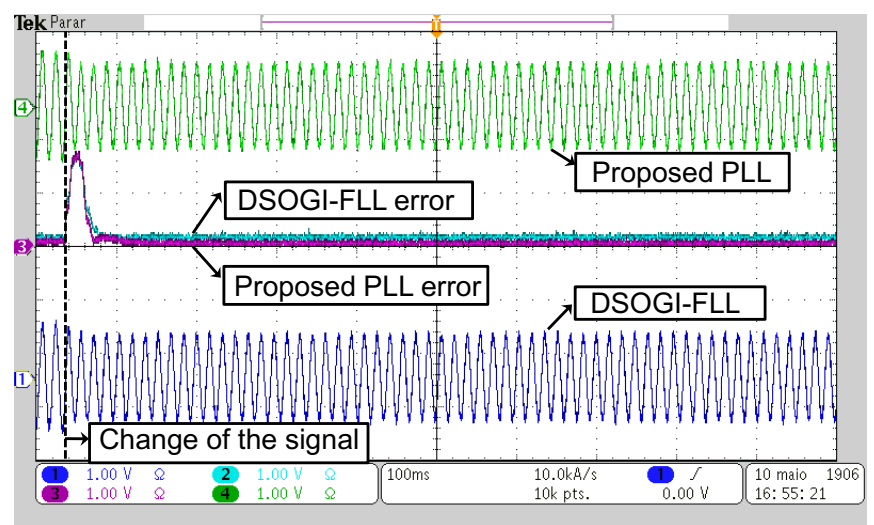

Fig. 16: Signal estimated by the proposed PLL, signal estimated by the DSOGI-FLL, error of the proposed PLL and error of the DSOGI-FLL.

\section{Conclusion}

In this paper, a new Three-Phase PLL (Phase-Locked Loop) algorithm was presented. One of the main characteristics of this PLL is the high immunity to subharmonics and interharmonics. In order to prove the effectiveness of the presented algorithm, were performed simulations through
PSCAD/EMTDC software and was assembled the PLL structure in HIL (Hardware-in-the-Loop) platform. The structure was submitted to a signal with the presence of distortions, such as subharmonics and interharmonics, under several operational conditions.

The results showed that the structure of the proposed PLL is able to estimate the synchronism angle, the frequency and the positive sequence component of the input signal accurately under stead state and dynamic conditions in three-phase systems. Thus, it can be used quite effectively in more intelligent control strategies for power converters.

\section{Acknowledgement}

The authors would like to thank the National Counsel of Technological and Scientific Development (CNPq) through the Project number 486948/2012-9, and Eletrobras Distribution Piaui for the financial support.

\section{References}

[1] H., Akagi, E. H., Watanabe, M., Aredes, "Instantaneous Power Theory and Applications to Power Conditioning", Wiley Inter-Science A John Willey \& Sons Inc., IEEE Press Series on Power Engineering, New Jersey, 2007.

[2] P. Rodriguez, R. Teodorescu, I. Candela, A. V., Timbus, M. Liserre, F. Blaabjerg, "New Positive-Sequence Voltage Detector for Grid Synchronization of Power Converters Under Faulty Grid Conditions", in Proc. IEEE Power Electronics Specialists Conference (PESC'06), Jun. 2006, pp. 1-6.

[3] F. Blaabjerg, R. Teodorescu, M. Liserre, A. V. Timbus, "Overview of Control and Grid Synchronization for Distributed Power Generation Sytems", IEEE Trans. Ind. Electronics, vol. 53, no. 5, Oct. 2006.

[4] M. K. Ghartemani, "A Novel Three-Phase Magnitude- PhaseLocked Loop System”, IEEE Trans. on Circuits and Systems - I: Regular Papers, vol. 53, no 8, pp. 1792- 1802, August 2006.

[5] S. Väliviita, "Zero-Crossing Detection of Distorted Line Voltages Using 1-b Measurements", IEEE Transactions on Industrial Electronics, vol. 46, no. 5, pp. 917-922, 1999.

[6] K.-J. Lee, J.-P.Lee, D. Shin, D.-W.Yoo, H.-J Kim, “A Novel Grid Synchronization PLL Method Based on Adaptive LowPass Notch Filter for Grid-Connected PCS", IEEE Transactions on Industiral Electronics, vol. 61, no. 1, pp. 292-301, 2014.

[7] M. S. Pádua, S. M. Deckmann, F. P. Marafão, D. Cólon, "Analysis of Different Synchronization Algorithms Based on PLL, RDFT, and Kalman Filter", 9th Brazilian Power Electronics Conference, Blumenau, 2007, pp. 181-186.

[8] D. R. Costa Jr., L. G. B. Rolim, M. Aredes, "Analysis and Software Implementation of a Robust Synchronizing Circuit Based on pq Theory", IEEE Transaction on Industrial Electronics, vol. 53, no. 6, pp. 1919-1926, 2006.

[9] M. Ciobotaru, R. Teodorescu, F. Blaabjerg, "A New- Single PLL Structure Based on Second Order Generalized Integrator", IEEE 37th Power Electronics Specialists Conference (PESC), pp. 1-6, Jeju, 2006.

[10] M. C. Benhabib, S. Saadate, "A New Robust Experimentally Validated Phase-Locked-Loop for Power Electronic Control”, EPE Journal, vol. 15, pp-36-47, Aug. 2005. 
[11] D. Sharmitha, D. Sundararajan, "Estimation of Harmonics and Interharmonics Using Phase-Locked Loop", Power, Energy and Control (ICPEC), 2013 International Conference, pp.514-519, 2013.

[12] L. Feola, R. Langella, A. Testa, "On the Effects of Unbalances, Harmonics and Interharmonics on PLL Systems", IEEE Transactions on Instrumentation and Measurement, vol. 62, no. 9, pp. 2399-2409, 2013.

[13] R. Langella, P. Marino, G. Raimondo, L. Rubino, N. Serbia, A. Testa, "On the Effects of Interharmonic Distorcion on Static Converters Controlled by Means of PLL Systems", Harmonics and Quality of Power (ICHQP), 2010 14th International Conference on, pp.1-6, 2010.

[14] Z. Hanzelka, A. Bien, "Guia de Aplicação de Qualidade de Energia", AGH University of Science and Technology, July, 2014.

[15] J. A. M. Neto, L. Lovisolo, B. W. França, M. Aredes, "Grid Synchronization System for Power Converters", Brazilian Power Electronics Conference (COBEP), pp. 749-755, 2009.

[16] M. C. Benhabib, S. Saadate, "A New Topology for a Modular Active Power Filter", IEEE International Symposium on Industrial Electronics (ISIE), pp. 827-832, 2005. 